\title{
An Optimized-Hierarchy-Aided Maximum Likelihood Detector for MIMO-OFDM
}

\author{
J. Akhtman and L. Hanzo \\ School of ECS, Univ, of Southampton, SO17 1BJ, UK. \\ Tel: +44-23-80-593 125, Fax: +44-23-80-593045 \\ Email: 1h@ccs.soton.ac.uk, http:/www-mobile.ccs.soton.ac.uk
}

\begin{abstract}
In this paper we propose a novel low-complexity Maximum Likelihood (ML) space-time detection method, which can be regarded as an advanced extension of the Complex Sphere Decoder (CSD). We demonstrate that as opposed to the previously published variants of the $\mathrm{CSD}$, the proposed technique may be employed in the so-called "over-loaded" scemario, where the number of transmit antennas exceeds that of the receive antemnas. The proposed method achieves the optimum performance of the ML detector even in heavily over-loaded scenarios, while the associated computational complexity is only moderately increased.
\end{abstract}

\section{INTRODUCTION}

The relevant information-theoretical analysis predicts [1] that substantial capacity gains are achievable in wireless communication systems employing a Multiple Input Multiple Output (MIMO) architecture using multiple antennas. Additionally, the employment of a MIMO architecture allows for the efficient exploitation of the spatial diversity available in a wireless MIMO environment, thus improving both the system's transmission integrity, as well as capacity. Orthogonal Frequency Division Multiplexing (OFDM) employed in conjunction with a Multiple-Input Multiple-Output (MIMO) architecture [2] constitutes a particularly attractive solution for future generations of wireless communication systems ${ }^{1}$.

On the other hand, the most potent space-time detection method found in the literature is constituted by the Sphere Decoder (SD) [3], which is capable of achieving the optimum performance of the Maximum Likelihood (ML) detector at a relatively low computational complexity. The SD was first proposed for employment in the context of space-time processing in [4]. The complex-valued version of the sphere decoder was proposed by Hochwald and ten Brink in [5]. The subject was further investigated by Damen et al. in [6]. Subsequently, an improved version of the Complex Sphere Decoder (CSD) was advocated by Pham et al. in [7]. However, to the best of our knowledge, all of the aforementioned SD schemes rely on the assumption of having a higher number of receive antennas than that of the transmit antennas, corresponding to $n_{\mathrm{r}}>m_{\mathrm{t}}$.

\footnotetext{
Acknowledgements: The work reported in this paper has formed part of the Wireless Enabling Techniques work area of the Core 3 Research Programme of the Virtual Centre of Excellence in Mobile and Personal Communications, Mobile VCE, www mobilevce.com, whose funding support, including that of EPSRC, is gratefully acknowledged. Fully detailed technical reports on this research are available to Industrial Members of Mobile VCF

${ }^{1}$ In the uplink of a multi-user OFDM system we assume OFDM-symbollevel synchronization between users.
}

By contrast, the high-complexity ML detector is free from this constraint, which becomes particularly restrictive in the up-link of a multi-user system, where the number of users supported becomes hard-limited by the number of receive antennas used at the base-station.

Against this background, in this paper we propose a novel Optimized Hierarchy $(\mathrm{OH})$ Reduced Search Algorithm (RSA)aided ML SDM detection method, which may be regarded as an advanced extension of the CSD method of [5] and [7]. More explicitly, the proposed method performs well in the so-called over-loaded scenario, where the number of transmit antennas $m_{\mathrm{t}}$ exceeds that of the receive antennas $n_{\mathrm{r}}$. A particularly interesting potential application is found in a MIMO scenario, where the system employs multiple transmit antennas and a single receive antenna. We demonstrate that the method proposed approaches the optimum performance of the ML detector even in heavily overloaded scenarios. Moreover, the associated computational complexity is only moderately increased even in heavily overloaded scenarios and it is almost independent of the number of receive antennas.

The rest of this paper is structured as follows. The system model as well as the principles of ML space-time detection are briefly outlined in Section II-A. A novel recursive ML detection technique is derived in Section II-B. Furthermore, the search optimization rules are described in Section II-C. The achievable performance of the proposed technique is quantified using extensive computer simulations and the corresponding results are provided in Section III, before offering our conclusions in Section IV.

\section{OPTIMIZED HIERARCHY REDUCED SEARCH ALGORITHM}

In this section we derive a technique, which we refer to as the Optimized Hierarchy Reduced Search Algorithm (OHRSA)-ML method designed for detecting constantenvelope modulation schemes, such as $M$-PSK, where the transmitted symbols $s$ satisfy the condition of $|s|^{2}=1, \forall s \in$ $\mathcal{M}$, and $\mathcal{M}$ denotes the set of $M$ complex-valued constellation points. However, it may be readily demonstrated that the method proposed can be generalized for arbitrary signal constellations, particularly for high-throughput multilevel modulation schemes, such as $M$-QAM. 


\section{A. Maximum Likelihood Detection}

The subcarrier-related MIMO-OFDM system model considered is given by [2]

$$
\mathrm{y}=\mathrm{Hs}+\mathrm{w},
$$

where $\mathrm{y}, \mathrm{w}$ and $\mathrm{x}$ denote the $n_{\mathrm{r}}$-dimensional received signal and AWGN sample vectors as well as the $m_{t}$-dimensional transmitted signal vector, respectively. Furthermore, $\mathbf{H}$ represents a $\left(n_{\mathrm{T}} \times m_{\mathrm{t}}\right)$-dimensional matrix of subcarrier-related CTF coefficients. Note that for the sake of brevity we omit the OFDM subcarrier and symbol indices $k$ and $n$. As outlined in [2], the ML SDM detector provides an $m_{\mathrm{t}}$-antenna-based estimated signal vector candidate $\hat{s}$, which maximizes the objective function defined as the conditional a posteriori probability $\mathcal{P}(\mathbf{s} \mid \mathrm{y}, \mathbf{H})$ over the set $\mathcal{M}^{m_{\mathrm{t}}}$ of legitimate solutions. More explicitly, we have

$$
\hat{\mathbf{s}}=\arg \max _{\mathbf{s} \in \mathcal{M}^{m_{\mathrm{t}}}} \mathcal{P}(\tilde{\mathbf{s}} \mid \mathbf{y}, \mathbf{H}),
$$

where $\mathcal{M}^{m_{t}}$ is the set of all possible $m_{t}$-dimensional candidate symbol vectors of the $m_{\mathrm{t}}$-antenna-based transmitted signal vector $\mathbf{s}$. Specifically, we have

$$
\mathcal{M}^{m_{\mathrm{t}}}=\left\{\check{\mathrm{s}}=\left(\check{s}_{1}, \cdots, \check{s}_{m_{\mathrm{t}}}\right)^{\mathrm{T}} ; \check{s}_{i} \in \mathcal{M}\right\} .
$$

Furthermore, it was shown in [2] that we have

$$
\mathcal{P}(\check{s} \mid \mathbf{y}, \mathbf{H})=A \exp \left[-\frac{1}{\sigma_{w}^{2}}\|\mathbf{y}-\mathbf{H} \check{s}\|^{2}\right],
$$

where $A$ is a constant, which is independent of any of the values $\left\{\breve{s}_{i}\right\}_{i=1, \cdots, m_{t}}$. Thus, it may be shown [2] that the probability maximization problem of Equation (2) is equivalent to the corresponding Euclidean distance minimization problem. Specifically, we have

$$
\hat{\mathbf{s}}=\arg \min _{\hat{\mathbf{s}} \in \mathcal{M}^{m_{\mathrm{t}}}}\|\mathrm{y}-\mathbf{H} \check{s}\|^{2},
$$

where the probability-based objective function of Equation (2) is substituted by the objective function determined by the Euclidean distance between the received signal vector $y$ and the corresponding product of the channel matrix $\mathbf{H}$ with the a priori candidate of the transmitted signal vector $\breve{s} \in \mathcal{M}^{m_{t}}$.

\section{B. Recurssive ML Detection}

Consequently, our detection method relies on the observation, which may be summarized in the following lemma.

Lemma 1: The ML solution of Equation (2) of a noisy linear problem described by Equation (1) is given by

$$
\hat{\mathrm{s}}=\arg \min _{\hat{\mathrm{s}} \in \mathcal{M}^{m_{\mathrm{t}}}}\left\{\|\mathbf{U}(\check{s}-\hat{\mathbf{x}})\|^{2}\right\},
$$

where $U$ is an upper-triangular matrix having positive realvalued elements on the main diagonal and satisfying

$$
\mathbf{U}^{\mathrm{H}} \mathbf{U}=\left(\mathbf{H}^{\mathrm{H}} \mathbf{H}+\sigma_{w}^{2} \mathbf{I}\right),
$$

while

$$
\hat{\mathbf{x}}=\left(\mathbf{H}^{\mathrm{H}} \mathbf{H}+\sigma_{u}^{2} \mathbf{I}\right)^{-1} \mathbf{H}^{\mathrm{H}} \mathbf{y}
$$

is the unconstrained MMSE estimate of the transmitted signal vector $\mathbf{s}$, which was derived in [2] .
Note 1: Observe that Lemma 1 imposes no constraints on the dimensions, or rank of the matrix $\boldsymbol{H}$ of the linear system described by Equation (1). This property is particularly important, since it enables us to apply our proposed detection technique to the scenario of over-loaded systems, where the number of transmit antenna elements exceeds that of the receive antema elements.

Note 2: As substantiated by Equation (5), it is sufficient to prove that the following minimization problems are equivalent

$$
\begin{aligned}
& \hat{\mathbf{s}}=\arg \min _{\hat{\mathbf{s}} \in \mathcal{M}^{m_{\mathrm{t}}}}\|\mathbf{y}-\mathbf{H} \mathbf{s}\|^{2} \\
& \Leftrightarrow \quad \hat{\mathbf{s}}=\arg \min _{\hat{\mathbf{s}} \in \mathcal{M}^{m_{\mathrm{t}}}}\|\mathbf{U}(\hat{\mathbf{s}}-\hat{\mathbf{x}})\|^{2} .
\end{aligned}
$$

Proof of Lemma 1: It is evident that in contrast to the matrix $\mathbf{H}^{\mathrm{H}} \mathbf{H}$, the matrix $\left(\mathbf{H}^{\mathrm{H}} \mathbf{H}+\sigma_{w}^{2} \mathbf{I}\right)$ of Equation (6) is always Hermitian and positively definite, regardless of the rank of the channel matrix $\mathbf{H}$ associated with the particular MIMO channel realization encountered. Consequently, $\left(\mathbf{H}^{\mathrm{H}} \mathbf{H}+\sigma_{w}^{2} \mathbf{I}\right)$ may be represented as the product of an upper-triangular matrix $U$ and its Hermitian adjoint matrix $U^{\mathrm{H}}$ using for example the Cholesky factorization method [8], [9, Cholesky Decomposition].

Let $\mathbf{U}$ be the matrix generated by the Cholesky decomposition of the Hermitian positive definite matrix $\left(\mathbf{H}^{\mathrm{H}} \mathbf{H}+\sigma_{w}^{2} \mathbf{I}\right)$ of Equation (7). More specifically, we have

$$
\mathbf{U}^{\mathrm{H}} \mathbf{U}=\left(\mathbf{H}^{\mathrm{H}} \mathbf{H}+\sigma_{w}^{2} \mathbf{I}\right)
$$

where $\mathbf{U}$ is an upper-triangular matrix having positive realvalued elements on its main diagonal.

Upon expanding the objective function of Equation (6) and subsequently invoking Equation (7) we obtain

$$
\begin{aligned}
& J(\tilde{\mathbf{s}})=\|\mathrm{U}(\overline{\mathrm{s}}-\hat{\mathrm{x}})\|^{2} \\
& =(\check{s}-\hat{\mathbf{x}})^{\mathrm{H}} \mathbf{U}^{\mathrm{H}} \mathbf{U}(\tilde{s}-\hat{\mathbf{x}}) \\
& =(\breve{s}-\hat{\mathbf{x}})^{\mathrm{H}}\left(\mathbf{H}^{\mathrm{H}} \mathbf{H}+\sigma_{w}^{2} \mathbf{I}\right)(\breve{s}-\hat{\mathbf{x}}) \\
& =\breve{s}^{\mathrm{H}}\left(\mathbf{H}^{\mathrm{H}} \mathbf{H}+\sigma_{w}^{2} \mathbf{I}\right) \check{\mathrm{s}}-\hat{\mathrm{x}}^{\mathrm{B}}\left(\mathbf{H}^{\mathrm{H}} \mathbf{H}+\sigma_{w}^{2} \mathbf{I}\right) \tilde{\mathrm{s}} \\
& -\check{s}^{\mathrm{H}}\left(\mathbf{H}^{\mathrm{H}} \mathbf{H}+\sigma_{w}^{2} \mathbf{I}\right) \hat{\mathbf{x}}+\hat{\mathbf{x}}^{\mathrm{H}}\left(\mathbf{H}^{\mathrm{H}} \mathbf{H}+\sigma_{w}^{2} \mathbf{I}\right) \hat{\mathbf{x}} \text {. }
\end{aligned}
$$

Furthermore, substituting Equation (8) into (12) yields

$$
\begin{aligned}
J(\check{\mathbf{s}}) & =\check{\mathbf{s}}^{\mathrm{H}} \mathbf{H}^{\mathrm{H}} \mathbf{H} \check{\mathbf{s}}-\mathbf{y}^{\mathrm{H}} \mathbf{H} \breve{s}-\breve{s}^{\mathrm{H}} \mathbf{H}^{\mathrm{H}} \mathbf{y} \\
& +\sigma_{w}^{2} \check{\mathbf{s}}^{\mathrm{H}} \check{\mathbf{s}}+\mathbf{y}^{\mathrm{H}} \mathbf{H}\left(\mathbf{H}^{\mathrm{H}} \mathbf{H}+\sigma_{w}^{2} \mathbf{I}\right)^{-1} \mathbf{H}^{\mathrm{H}} \mathbf{y} \\
& =\|\mathbf{y}-\mathbf{H} \check{\mathbf{s}}\|^{2}+\underbrace{\sigma_{w}^{2} \check{\mathbf{s}}^{\mathrm{H}} \breve{\mathbf{s}}+\mathbf{y}^{\mathrm{H}}\left(\mathbf{H}\left(\mathbf{H}^{\mathrm{H}} \mathbf{H}+\sigma_{w}^{2} \mathbf{I}\right)^{-1} \mathbf{H}^{\mathrm{H}}-\mathbf{I}\right) \mathbf{y}}_{\psi} .
\end{aligned}
$$

Observe that in the case of a system employing a constantenvelope modulation scheme, such as M-PSK, where we

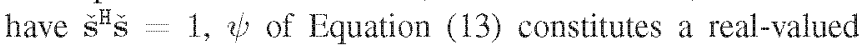
scalar and its value does not depend on the argument $s$ of the minimization problem formulated in Equation (6). Consequently, the minimization of the objective function $J(\mathbf{s})$ of Equation (13) can be reduced to the minimization of the term $\| \mathrm{y}-\mathrm{Hx}) \|^{2}$, which renders it equivalent to the minimization problem of Equation (9). This completes the proof. 
Using Lemma 1, in particular the fact that the matrix $U$ is an upper-triangular matrix, the objective function $J(s)$ of Equation (13) may be reformulated as follows

$$
\begin{aligned}
J(s) & =\|\mathbf{U}(\dot{s}-\hat{\mathbf{x}})\|^{2} \\
& =(s-\hat{\mathbf{x}})^{\mathrm{H}} \mathbf{U}^{\mathrm{H}} \mathbf{U}(\mathbf{s}-\hat{\mathbf{x}}) \\
& =\sum_{i=1}^{m_{\mathrm{t}}}\left|\sum_{j=i}^{m_{\mathrm{t}}} u_{i j}\left(\check{s}_{j}-\hat{x}_{j}\right)\right|^{2}=\sum_{i=1}^{m_{\mathrm{t}}} \phi_{i}\left(\check{s}_{i}\right),
\end{aligned}
$$

where $J(\breve{s})$ and $\dot{\phi}_{i}\left(\bar{s}_{i}\right)$ are positive real-valued cost and subcost functions, respectively. Elaborating a little further, we have

$$
\begin{aligned}
\phi_{i}\left(\breve{s}_{i}\right) & =\left|\sum_{j=i}^{m_{\mathrm{t}}} u_{i j}\left(\check{s}_{j}-\hat{x}_{j}\right)\right|^{2} \\
& =\mid u_{i i}\left(\check{s}_{i}-\hat{x}_{i}\right)+\underbrace{\left.\sum_{j=i+1}^{m_{\mathrm{t}}} u_{i j}\left(\check{s}_{j}-\hat{x}_{j}\right)\right|^{2}}_{a_{i}} .
\end{aligned}
$$

Note that the term $a_{i}$ is a complex-valued scalar, which is independent of the specific symbol value $s_{i}$ of the thelement of the a priori candidate signal vector $s$.

Furthermore, let $J_{i}\left(\check{s}_{i}\right)$ be a Cumulative Sub-Cost (CSC) function recursively defined as

$$
\begin{aligned}
J_{m_{\mathrm{t}}}\left(\check{s}_{m_{\mathrm{t}}}\right) & =\phi_{m_{\mathrm{t}}}\left(\check{s}_{m_{\mathrm{t}}}\right)=\left|u_{m_{\mathrm{t}} m_{\mathrm{t}}}\left(\check{s}_{m_{\mathrm{t}}}-\hat{x}_{m_{\mathrm{t}}}\right)\right|^{2} \\
J_{i}\left(\check{s}_{i}\right) & =J_{i+1}\left(\check{s}_{i+1}\right)+\phi_{i}\left(\check{s}_{i}\right), \quad i=1, \cdots, m_{\mathrm{t}}-1
\end{aligned}
$$

where we define the candidate subvector as $s_{i}=$ $\left[\breve{s}_{i}, \cdots, \breve{s}_{m_{\mathrm{t}}}\right]$. Clearly, $J_{i}\left(\check{s}_{i}\right)$ exhibits the following properties

$$
\begin{aligned}
J(\breve{s}) & =J_{1}\left(\check{s}_{1}\right)>J_{2}\left(\check{s}_{2}\right)>\cdots>J_{m_{\mathrm{t}}}\left(\check{s}_{m_{\mathrm{t}}}\right)>0 \\
J_{i}\left(\breve{s}_{i}\right) & =J_{i}\left(\left\{\check{s}_{j}\right\}, j=i, \cdots, m_{\mathrm{t}}\right)
\end{aligned}
$$

for all possible realizations of $\hat{\mathrm{x}} \in \mathbb{C}^{m_{\mathrm{t}}}$ and $\breve{\mathrm{s}} \in \mathcal{M}^{m_{\mathrm{t}}}$, where the space $\mathbb{C}^{m_{t}}$ contains all possible unconstrained MMSE estimates $\hat{x}$ of the transmitted signal vector $\mathrm{s}$.

Equations (17a) and (17b) enable us to employ a highly efficient reduced-complexity search algorithm, which decreases the number of objective function evaluations of the minimization problem outlined in Equation (6) to a small fraction of the set $\mathcal{M}^{m_{t}}$. This reduced-complexity search algorithm is oulined in the next section.

\section{Search Strategy}

Firstly, we commence the recursive search process with the evaluation of the CSC function value $J_{m_{t}}\left(\breve{s}_{m_{t}}\right)$ of Equation (16a). Secondly, at each recursive step $i$ of the search algorithm proposed we stipulate a series of hypotheses concerning the value of the $M$-ary transmitted symbol $s_{i}$ associated with the th transmit antema element and subsequently calculate the conditioned sub-cost function $J_{i}\left(s_{i}\right)$ of Equation $(16 \mathrm{~b})$, where $s_{i}=\left(\breve{s}_{i}, \cdots, \breve{s}_{m_{+}}\right)^{\mathrm{T}}$ denotes the subvector of the $m_{\mathrm{t}}$-antennabased candidate vector $\$$ comprising only the indices higher than or equal to $i$. Furthermore, for each tentatively assumed value of $s_{i}$ we execute a successive recursive search step $i-1$, which is conditioned on the hypotheses made in all preceding recursive steps $j=i, \cdots, m_{t}$. As substantiated by Equations (15) and (16b), the value of the CSC function $J_{i}\left(s_{i}\right)$ is dependent only on the values of the elements $\left\{\ddot{s}_{j}\right\}_{j=i}, \cdots, m_{t}$ of the a priori candidate signal vector $s$, which are hypothesized from from step $j=m_{\mathrm{t}}$ up to the present step $i$ of our recursive process. Upon each arrival at the index $\dot{i}=1$ of the recursive process, a complete candidate vector $s$ is hypothesized and the corresponding value of the cost function $J(5)$ formulated in Equation (14) is evaluated.

Observe that the recursive hierarchical search procedure described above may be employed to perform an exhaustive search through all possible values of the transmitted signal vector $s$ and the resultant search process is guaranteed to arrive at the ML solution $\hat{s}$, which minimizes the value of the cost function $J(s)$ of Equation (14). Fortunately however, as opposed to other ML search schemes, the search process described above can be readily optimized, resulting in a dramatic reduction of the associated computational complexity. Specifically, the potential optimization complexity gain accrues from the fact that most of the hierarchical search branches can be discarded at an early stage of the recursive search process. The corresponding optimization rules proposed may be outlined as follows.

Rule 1: We reorder the system model of Equation (1) as suggested in [10]. Specifically, we apply the best-first detection strategy outlined in $[2$, pp.754-756], which implies that the transmitted signal vector components are detected in the decreasing order of the associated chamnel quality. As it was advocated in [2, pp.754-756], the quality of the channel associated with the the element of the transmitted signal vector $s$ is determined by the norm of the $i$ th column of the channel matrix $\boldsymbol{H}$. Consequently, for the sake of applying the bestfirst detection strategy, the columns of the channel matrix $\mathbf{H}$ are sorted in the increasing order of their norm. Thus, the resultant, columm-reordered channel matrix $\mathbf{H}$ complies with the following criterion

$$
\left\|(\mathbf{H})_{1}\right\|^{2} \leq\left\|(\mathbf{H})_{2}\right\|^{2} \leq \cdots \leq\left\|(\mathbf{H})_{m_{t}}\right\|^{2},
$$

where $(\mathbf{H})_{i}$ denotes the th column of the channel matrix $\mathbf{H}$. Note that the elements of the transmitted signal vector s are reordered correspondingly, but their original order has to be reinstated in the final stage of the detection process.

Rule 2: At each recursive detection step $i=m_{t}, \cdots, 1$, the potential candidate values $\left\{c_{m}\right\}_{m=1}, \cdots, M \in \mathcal{M}$ of the transmitted signal component $s_{i}$ are considered in the increasing order of the corresponding value of the sub-cost function $\phi_{i}\left(s_{i}\right)=\phi_{i}\left(c_{m}, \ddot{s}_{i+1}\right)$ of Equation (15), where we have

$$
\phi_{i}\left(c_{1}, \check{s}_{i+1}\right)<\cdots<\phi_{i}\left(c_{m}, \check{s}_{i+1}\right)<\cdots \phi_{i}\left(c_{M}, \check{s}_{i+1}\right)
$$

and according to Equation (15)

$$
\begin{aligned}
\phi_{i}\left(c_{m}, \check{s}_{i+1}\right) & =\left|u_{i i}\left(c_{m}-\hat{x}_{i}\right)+a_{i}\right|^{2} \\
& =u_{i i}\left|c_{m}-\hat{x}_{i}+\frac{a_{i}}{u_{i i}^{2}}\right|^{2} .
\end{aligned}
$$

Consequently, the more likely candidates $c_{m}$ of the th element of the transmitted signal vector $s$ are examined first. Observe that the sorting criterion of Equation (19) may also 
be interpreted as a biased Euclidean distance of the candidate constellation point $c_{m}$ from the unconstrained MMSE estimate $\hat{x}_{i}$ of the transmitted signal component $s_{i}$.

Rule 3: We define a cut-off value of the cost function $J_{\min }=\min \{J(s)\}$ as the minimum value of the total cost function obtained up to the present point of the search process. Consequently, at each arrival at step $i=1$ of the recursive search process, the cut-off value of the cost function is updated as follows

$$
J_{\min }=\min \left\{J_{\min }, J(\mathbf{s})\right\} .
$$

Rule 4: Finally, at each recursive detection step $i$, only the high probability search branches corresponding to the highly likely symbol candidates $c_{m}$ resulting in particularly low values of the CSC function obeying $J_{i}\left(c_{m}\right)<J_{\min }$ are pursued. Furthermore, as follows from the sorting criterion of the optimization Rule 2, as soon as the inequality $J_{i}\left(c_{m_{i}}\right)>$ $J_{\min }$ is encountered, the search loop at the $i$ th detection step is discontinued.

An example of the search tree generated by the algorithm described above is depicted in Figure 1. The search trees shown correspond to the scenario of using BPSK modulation and employing $m_{\mathrm{t}}=n_{\mathrm{r}}=3$ antenna elements at both the transmitter and the receiver. A scenario of encountering the average SNR of $10 \mathrm{~dB}$ is considered. Each evaluation step of the search procedure is denoted by an elliptical node. The label associated with each node indicates the order of visitation, as well as the corresponding value of the CSC function $J_{i}\left(\breve{s}_{i}\right)$ formulated in Equation (16), as seen in the brackets. As suggested by the fact that BPSK modulation is considered, at each recursive step $i$, two legitimate search branches are possible. The branches corresponding to the two legitimate values $\tilde{s}_{i}=-1$ and 1 are indicated using the dashed and solid edges and nodes, respectively. Firstly, Figure 1(a) illustrates an unoptimized exhaustive recursive search. Secondly, Figure 1(b) depicts the search tree obtained by performing exhaustive recursive search optimized by invoking Rules 1 and 2 described above. Finally, Figure 1(c) depicts the corresponding reduced search tree obtained by executing OHRSA, i. e. invoking Rules 1-4 outlined above. It should be noted that the convergence rate of the algorithm proposed is particularly high at high SNR values. In the case of encountering low SNR values, the convergence rate decreases. Nevertheless, the associated computational complexity is substantially lower than that associated with an exhaustive ML search. The pseudo-code summarizing the recursive implementation of the OHRSA-ML detector proposed is depicted in Algorithm 1.

\section{Simulation RESULTS}

Our simulations were performed in the base-band frequency domain. The OFDM system considered utilises 128 QPSKmodulated orthogonal subcarriers. For forward error correction (FEC) we use $\frac{1}{2}$-rate turbo coding [11] employing two constraint-length $K=3$ Recursive Systematic Convolutional (RSC) component codes and the standard 124-bit WCDMA UMTS turbo code interleaver of [12]. The octally represented RCS generator polynomials of $(7,5)$ were used. We assume a

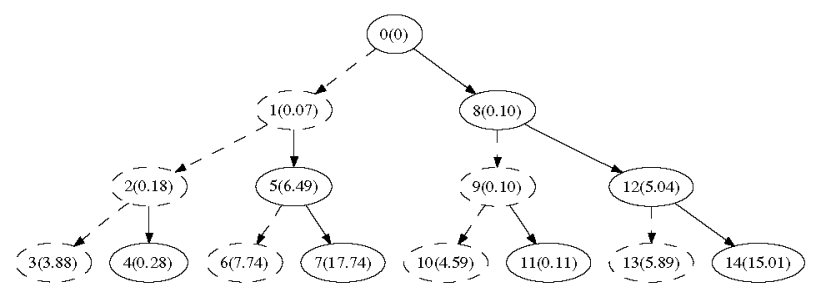

(a)

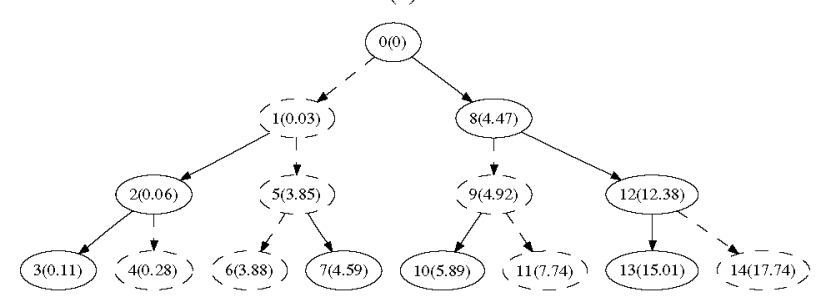

(b)

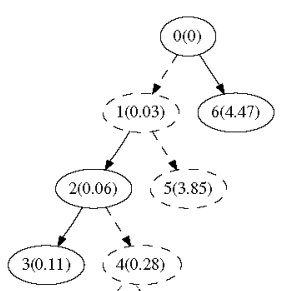

$(0)$

Fig. 1. Examples of a search tree formed by the OHRSA-ML SDM detector in the scenario of a system employing BPSK modulation, $m_{\mathrm{t}}=n_{\mathrm{T}}=3$ transmit and receive antennas and encountering average SNRs of $10 \mathrm{~dB}$. The labels indicate the order of visitation, as well as the corresponding value $J_{i}\left(\mathrm{~s}_{i}\right)$ of the CSC function, as seen in the brackets.

9-tap CIR Rayleigh-fading multipath channel and stipulate the assumption of perfect channel knowledge, where the knowledge of the frequency-domain subcarrier-related coefficients $H[n, k]$ is deemed to be available in the receiver.

Figure 2 characterizes the achievable performance of the MIMO-OFDM system employing the OHRSA-ML detector proposed in the fully loaded system scenario, where the number of the transmit antenna elements $m_{\mathrm{t}}$ is equal to that of the receiver antennas $n_{\mathrm{r}}$.

Additionally, Figure 3 characterizes the MIMO-OFDM system employing the OHRSA-ML detector and having a constant number of $n_{\mathrm{r}}=4$ receive antennas in terms of its ability to detect the multiplexed signals arriving from various numbers of users. Specifically, we aim for exploring the performance of the OHRSA-ML detector in the overloaded system scenario, where the number of users exceeds that of the receive antennas and thus we have $m_{\mathrm{t}}>n_{\mathrm{r}}$. Figure 3 quantifies the achievable BER performance of the MIMO-OFDM system employing the OHRSA-ML detector as a function of the average $E_{b} / N_{0}$ encountered. We can see that as opposed to the conventional CSD detector [5], the OHRSA-ML detector proposed exhibits a good performance both when we have $m_{\mathrm{t}} \leq n_{\mathrm{r}}$, as well as in the overloaded system scenario, when the number of transmit antennas exceeds that of the receive antennas, i.e. when we have $m_{\mathrm{t}}>n_{\mathrm{r}}$.

\section{CONCLUSION}

We proposed a novel OHRSA-ML space-time detector, which may be regarded as an advanced extension of the 


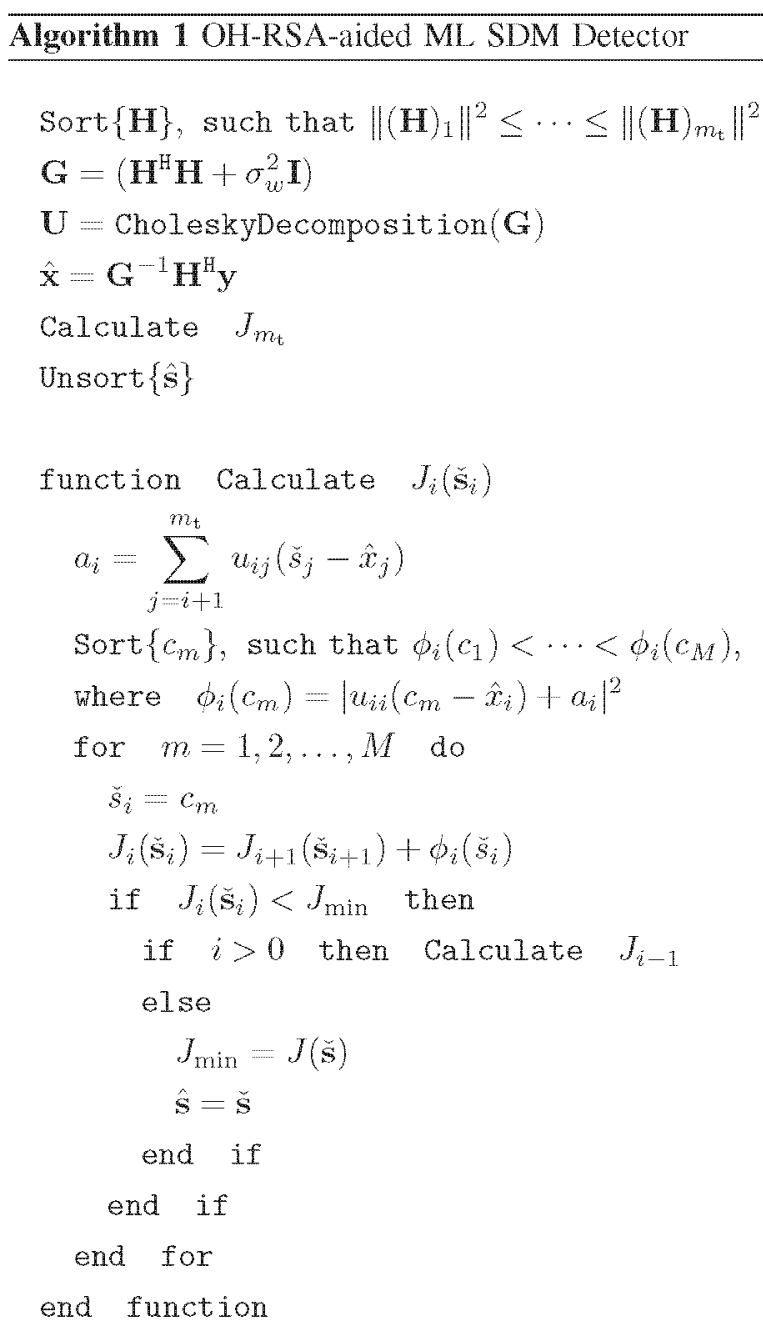

CSD method. We demonstrated that the MIMO-OFDM system employing the OHRSA-ML detector proposed is capable of achieving the near optimum ML performance in the overloaded scenario, where the number of transmit antemas exceeds that of the receive antennas.

\section{REPERENCES}

[1] A. Goldsmith, S. A. Jafar, N. Jindal, and S. Vishwanath, "Capacity limits of MIMO channels," IEEE Joumal on Selected Areas in Communicathons, vol. 21, no. 5, pp. 684-702. June 2003.

[2] L. Hanzo, Münster, B. Choi, and T. Keller, OFDM and MC-CDMA for Broadband Multi-tiser Conmunications, WLANs and Broadcasting. John Wiley and IEEE Press, 2003, 992 pages.

[3] U. Fincke and M. Pohst, "Improved method for calculating vector of short length in a lattice, including a complexity analysis," Math. Comput. vol. 44, pp. 463-471, April 1985.

[4] M. O. Damen, A. Chkeif, and J.-C. Belfiore, "Lattice code decoder for space-time codes," IEEE Commun, Letters, pp. 161-163, May 2000.

[5] B. M. Hochwald and S. ten Brink, "Achieving near-capacity on a multiple-antenna channel," IEEE Transactions on Communications, vol. 51, no, 3, pp. 389-399, March 2003.

[6] M. O. Damen, H. E. Gamal, and G. Caier, "On maximum-likelihood detection and the search for closest latice point," IEEE Transactions on Information Theory, vol. 49, no. 10, pp. 2389-2402, October 2003.

[7] D. Pham, K. R. Pattipati, P. K. Willet, and J. Luo, "An improved complex sphere decoder for V-BLAST Systems." IEEE Signal Processing Letters, vol. 11 , no. 9, pp. 748-751, September 2004.

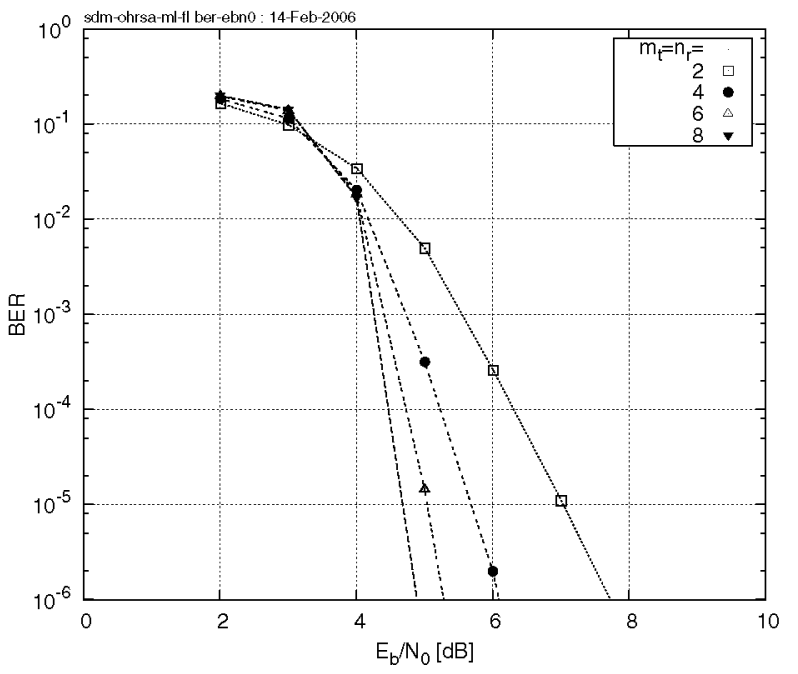

Fig. 2. Bit Erron Rate exhibited by the rate- $\frac{1}{2}$ urbo-coded MMO. OIDM system employing the enhanced CSD-aided SDM detector and $m_{t}=$ $n_{\mathrm{r}}=2,4,6$ and 8 transmit and receive antennas. The abscissa represents the average $E_{b} / N_{0}$.

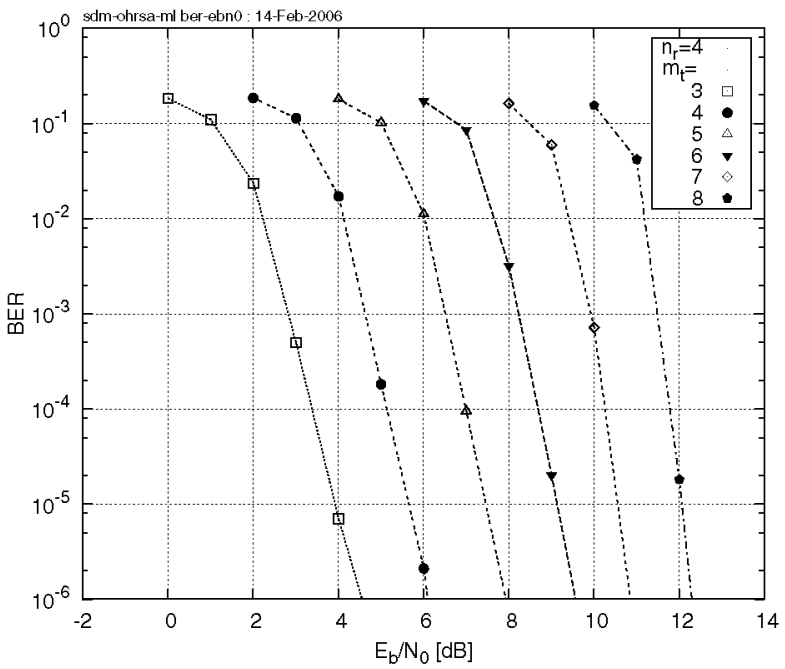

Fig. 3. Bit Error Rate exhibited by the rate- $\frac{1}{2}$ turbo-coded MIMOOFDM system employing the enhanced CSD-aided SDM detector and $m_{t}=$ $3,4, \cdots, 8$ transmit antemnas, as well as $n_{\mathrm{r}}=4$ receive antennas. The abscissa represents the average $E_{b} / N_{0}$.

[8] J. E. Gentle, Numerical Linear Algebra for Applications in Statistics. Berlin: Springer-Verlag, 1998.

[9] E. W. Weisstein. "MathWorld - A Wolfram Web Resource," hitp://mathworld.wolfram.com.

[10] M. K. Varanasi, "Decision feedback multiuser detection: A systematio approach," IEEE Transactions on Information Theory, vol. 45, pp. 219 240. January 1999.

[11] L. Hanzo, T. H. Liew, and B. L. Yeap, Turbo Coding. Turbo Equalisation and Space-Time Coding. Chichester, UK; Piscataway, NI, USA: John Wiley and TEEE Press, 2002, 766 pages. (For detailed contents, please refer to hitp://www-mobile.ecs.soton.ac.uk.).

[12] H. Holma and A. Toskala, Eds., WCDMA for UMTS : Radio Access for Third Generation Mobile Conmunications. John Wiley and Sons, Ltd. 2000 . 